\title{
El uso de weblogs en la educación superior y su evaluación mediante rúbrica
}

Judit Tabernaa ${ }^{a}$, Santiago Domínguez-Garcíab y María Isabel García-Planas ${ }^{c}$

a Universitat Politècnica de Catalunya, Judit.taberna@gmail.com buniversitat Rovira i Virgili

soyelsanti31@gmail.com, y c Universitat Politècnca de Catalunya, maria.isabel.garcia@upc.edu.

\begin{abstract}
Weblogs use is becoming more common among university teachers so, it becomes increasingly necessary to define a good evaluation system. The evaluation criteria of a blog should consider the operation, appearance, evidence of the work done in both quality and quantity indicators for reflection and the level of participation and collaboration of students.

Considering these aspects and to respond to those needs, we have chosen a rubric as a methodology for evaluating blogs.

For the specific topic of blogs used in the subject of linear algebra course in ETSEIB of the Universitat Politècnica de Catalunya, two rubrics, one for self-assessment and peer assessment, and the second for evaluating student work by the teacher are proposed. This second rubric consists of 3 subrubrics, one for analyzing the formal part of the student blog (structure, spelling, language level, etc.) and the other two for evaluating projects that students must perform.
\end{abstract}

Keywords: weblog, innovation, rubric, higher education, teaching resources,

\footnotetext{
Resumen

El uso de weblogs se va haciendo más habitual entre los docentes universitarios por lo que, se hace cada vez más necesario definir un buen sistema de evaluación. Los criterios de evaluación de un blog deben tener en cuenta la operatividad, la apariencia, la evidencia del trabajo realizado tanto en calidad como cantidad, los indicadores de reflexión y el nivel de participación y colaboración de los estudiantes.

Teniendo en cuenta estos aspectos y para responder a esas necesidades hemos elegido la rúbrica como metodología para la evaluación de blogs.
} 
Para el tema específico de blogs utilizados en la asignatura álgebra lineal cursada en la ETSEIB de la Universidad Politécnica de Cataluña, se proponen dos rúbricas, una para la auto-evaluación y evaluación por pares, y la segunda para evaluar el trabajo del estudiante por parte del docente. Esta segunda rúbrica consiste en 3 sub-rúbricas, una para analizar la parte formal del blog del alumno (estructura, ortografía, nivel de idioma, entre otros) y las otras dos para evaluar los proyectos que los estudiantes deben realizar.

Palabras clave: weblog, innovación, rúbrica, educación superior, recursos didácticos,

\section{Introducción}

Es bien reconocida la transformación que ha experimentado la Universidad por la creación del Espacio Europeo de Educación Superior (EEES). Uno de los retos es el centrar los procesos de enseñanza y aprendizaje en el aprendizaje autónomo de competencias genéricas y específicas, no sólo para afrontar el futuro profesional del estudiante sino para afrontar el futuro en general.

Para hacer frente a este reto se hace necesario adoptar nuevos roles tanto por parte de los docentes como por parte de los estudiantes, introduciendo otras metodologías, en particular las relacionadas con las TIC.

El weblog se está consolidando como una herramienta de aprendizaje en el aula y desde el curso 2014-15 y como prueba piloto, se ha incluido como propuesta metodológica para la asignatura de "Álgebra lineal" de los Grados en "Enginyeria en Tecnologies Industrials", en "Enginyeria Química” y en "Enginyeria de Materials” que se imparten en l'Escola Tècnica Supeior d'Enginyeria Industrial de Barcelona de la Universitat Politècnica de Catalunya.

Para conocer los resultados y la eficacia de un proceso de enseñanza-aprendizaje se debe realizar una actividad evaluativa. Sin la evaluación sería difícil conocer los resultados y la eficiencia de la acción docente y de los recursos de enseñanza utilizados, que permitan proponer correcciones y mejoras sobre la actividad evaluada.

Bloom, Madaus, y Hastings (1981) afirman que para mejorar en el aprenendizaje la evaluación es necesaria ya que ayuda a consolidarlo.

Se puede definir la evaluación como "un proceso sistemático, continuo e integral destinado a determinar hasta que punto fueron logrados los objetivos educacionales previamente determinados” (A.A. Hernández Sánchez 2010).

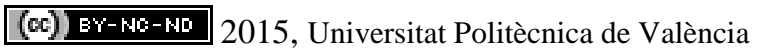


La evaluación ha de ofrecer una retroalimentación, ya que además de una calificación numérica ha de aportar información sobre las dificultades y errores de los participantes y así poder mejorar o trabajar en aquellos aspectos.

Teniendo en cuenta estos aspectos y para responder a esas necesidades hemos elegido la rúbrica o esquema de puntuación descriptiva, como metodología para la evaluación de weblogs.

Para el tema específico de weblogs utilizados en la prueba piloto que hemos puesto en marcha, se proponen dos rúbricas, una para el estudiante y otra para el docente.

La utilización de rúbricas se está extendiendo cada vez más, y se está convirtiendo en una práctica habitual en las aulas universitarias debido a que fomenta la implicación de los alumnos en sus procesos de formación y evaluación y proporciona un recurso que permite la autoevaluación del alumno y la evaluación entre pares.

\section{Objetivos}

La evaluación adquiere cada día mayor relevancia en los procesos y acciones de enseñanzaaprendizaje, que afecta al proceso que se desarrolla con el fin de lograr los objetivos previamente fijados. Siempre hay una necesidad de evaluar las habilidades de formación adquiridas para, de esta forma, saber si se han logrado los objetivos de aprendizaje. El uso de weblogs se va haciendo más habitual entre los docentes universitarios por lo que, se hace cada vez más necesario definir un buen sistema de evaluación. Los criterios de evaluación de un blog deben tener en cuenta la operatividad, la apariencia, la evidencia del trabajo realizado tanto en calidad como cantidad, los indicadores de reflexión y el nivel de participación y colaboración de los estudiantes.

Teniendo en cuenta todos estos aspectos y para responder a esas necesidades se ha elegido la creación de rúbricas para la evaluación del weblog. Se recuerda que las rúbricas de puntuación son sistemas de puntuación descriptivos, desarrolladas por profesores u otros evaluadores para orientar el análisis de los productos o procesos de esfuerzos de los estudiantes. Las rúbricas son tablas de doble entrada en las cuales se enumeran los criterios de evaluación de manera detallada y gradual, para poder ponderar de la forma más objetiva posible las competencias de los estudiantes, ya que ellos pueden valorar y revisar su propio trabajo antes de entregarlo.

\subsection{El weblog para un curso de Álgebra lineal}

El importante avance de las Tecnologías de la Información y Comunicación (TIC) ha conllevado un cambio en el mundo de la educación en particular con las herramientas

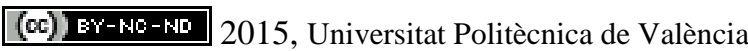


disponibles que facilitan el proceso de enseñanza-aprendizaje. Una herramienta en el contexto de las TIC es el uso del weblog.

El Álgebra lineal aporta, al perfil del ingeniero, la capacidad para desarrollar un pensamiento lógico, heurístico y algorítmico al modelar fenómenos de naturaleza lineal y resolver problemas, por lo que es un asignatura que induce el estudio reflexivo además del cuantitativo que se supone a toda asignatura de matemáticas. Dadas pues, las características de la asignatura, decidimos llevar a cabo una prueba piloto durante el curso académico 2014-15 que permitiera valorar la viabilidad de la utilización de weblogs como instrumento de aprendizaje para la asignatura de Álgebra lineal.
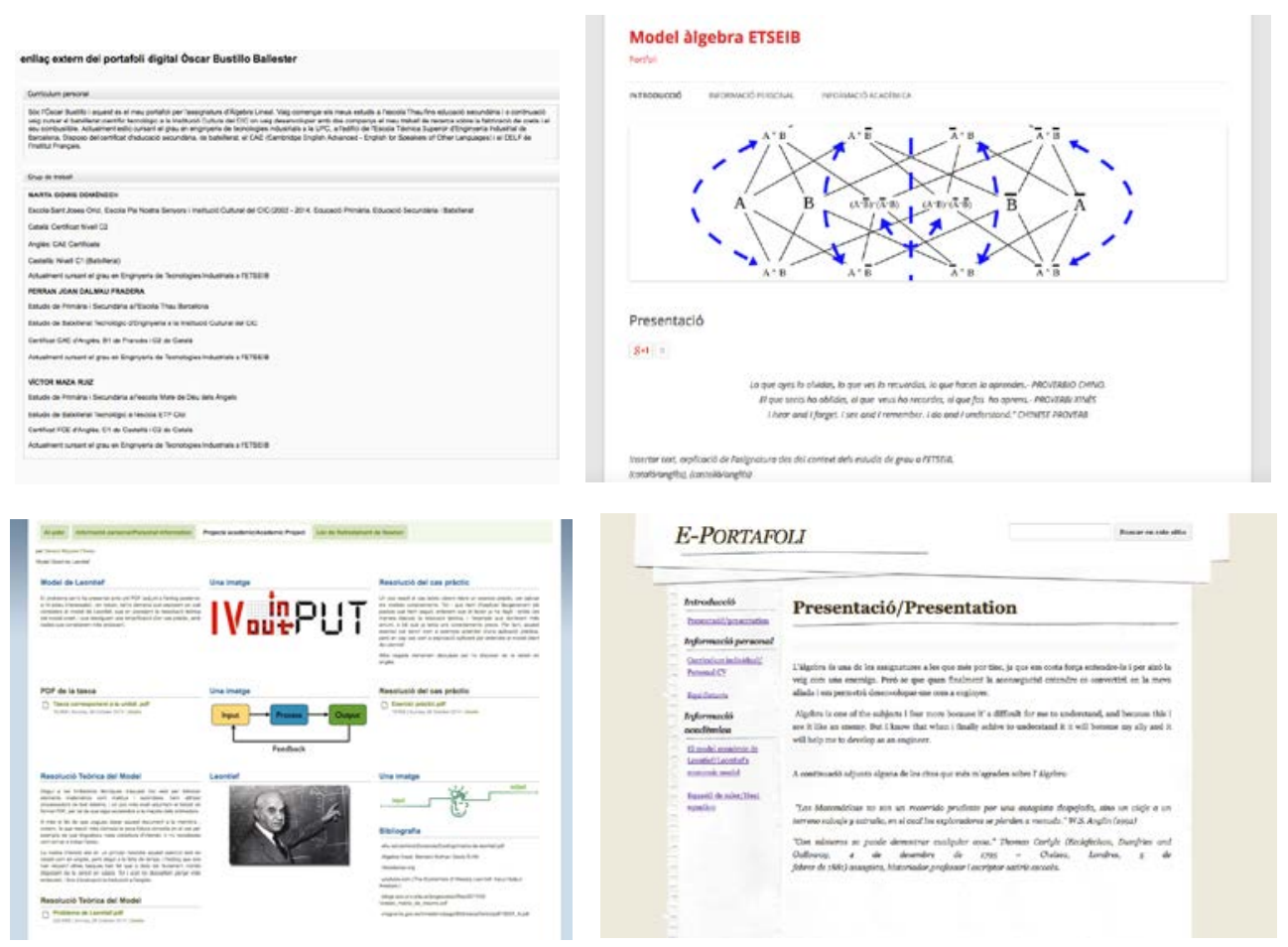

Fig1. Ejemplos plataformas, de arriba abajo y de izquierda a derecha: Exabis, Wordpress, Mahara y Google Sites

Los estudiantes del primer año de la Universidad en la ETSEIB han estado utilizando diferentes plataformas para la creación de weblogs de soporte a su aprendizaje en la materia de Álgebra Lineal. Concretamente las plataformas utilizadas son Moodle y Mahara (Mahoodle) plataforma integrada, así como el uso simultáneo de plataformas Exabis dentro de Moodle, Google Sites (entorno Google) y Wordpress dentro del servidor de la UPC. (Ver Fig.1). Según las encuestas realizadas a los estudiantes las plataformas en las que prefieren trabajar son Google Sites y Wordpress. 
Para que los estudiantes creasen su propio weblog ha sido esencial la definición clara y concisa de los objetivos que deseamos que los estudiantes alcancen, así como los temas que se consideran básicos para superar los objetivos.

En el contexto de la enseñanza y el aprendizaje de la asignatura de álgebra lineal, el weblog se está utilizando como un repositorio de experiencias de aprendizaje a través del cual los profesores y los estudiantes trabajan las actividades de la asignatura desde Internet.

Durante la primera semana de curso, se explica a los alumnos en que consiste un weblog y que uso se le va a dar, así como los proyectos que los estudiantes tienen que realizar tanto de forma individual como en grupo. Así como toda la planificación del cuatrimestre estructurada por semanas.

\subsection{La rúbrica como herramienta de evaluación}

Una rúbrica es un instrumento de calificación que se utiliza para evaluar de forma objetiva el aprendizaje de los estudiantes. Moskal en (Moskal (2000)) describe la rúbrica como un esquema con una puntuación descriptiva desarrollada por profesores u otros evaluadores para guiar el análisis de la producción o proceso realizado por los alumnos en un trabajo o práctica.

\subsubsection{Rúbrica del profesor}

La rúbrica del profesor evalúa las dos tareas encomendadas a los estudiantes así como la estructura y la relación entre las plantillas.

Se han planteado una serie de preguntas reflexivas para la realización de la rúbrica que se describen a continuación:

Diseño gráfico, multimedia, navegación, legibilidad

Citas y bibliografía

Calidad de la escritura y corrección

Ejercicio de compresión, análisis de datos, incógnitas, el modelado, la resolución y la verificación de la solución.

Reflexión crítica sobre el trabajo realizado

El uso de dos idiomas

La coherencia entre la plantilla y el resultado propuesto. 


\subsubsection{Rúbrica del estudiante}

Las rúbricas son una herramienta valiosa para que los estudiantes hagan una autoevaluación. Debido a que las rúbricas no sólo consisten en una lista de criterios para el éxito, sino que también proporcionan descripciones de los niveles de rendimiento. Los estudiantes son capaces de utilizarlas para monitorear el progreso durante la ejecución de una tarea o actividad a evaluar.

Se han planteado una serie de preguntas reflexivas para la realización de la rúbrica que se describen a continuación:

Su trabajo tiene coherencia entre la plantilla y el resultado propuesto.

Su blog se ha escrito en, al menos, dos idiomas con buen nivel de competencia.

Ha utilizado suficientes y adecuados recursos bibliográficos.

Los artículos han sido seleccionados adecuadamente.

Su blog es legible

Su blog tiene un diseño gráfico coherente.

En la realización de las tareas se han analizado los datos de forma adecuada

Se ha modelado correctamente el caso propuesto

Ha resuelto los problemas

Ha verificado las soluciones

Ha hecho una reflexión crítica de su trabajo

\section{Desarrollo}

El caso de estudio es el conjunto de estudiantes de primer curso de la asignatura de “Álgebra lineal” de los tres Grados de Ingeniería (Enginyeria en Tecnologies Industrials, Enginyeria Química y Enginyeria de Materials) que se imparten en l'Escola Tècnica Supeior d'Enginyeria Industrial de Barcelona de la Universitat Politècnica de Catalunya. En dicho curso participan 680 alumnos repartidos en 10 grupos de alrededor de 60 a 70 alumnos por grupo y todos ellos trabajan individualmente en sus blogs. Al mismo tiempo, los estudiantes realizan tareas de álgebra lineal en grupos de cuatro alumnos constituidos con anterioridad y estos subgrupos se han mantenido a lo largo de todo el curso.

En la creación de la rúbrica, primero analizamos cuáles son los criterios de evaluación a considerar para un curso de álgebra lineal de primer año de grado de ingeniería y tener en cuenta el conjunto de habilidades necesarias para demostrar que han adquirido las 
competencias establecidas por la Universidad en el curso a evaluar. En la medida en que se trata de un curso de matemáticas, no hay que olvidar los siguientes niveles de calidad que corresponden a resolución, razonamiento y demostraciones, comunicación, conexiones y representación, de excelente a pobre, para una tarea específica.

La elaboración y resolución de los proyectos a través del weblog contabilizan un 30\% de la nota global de curso cuya calificación se obtiene a través de rúbricas.

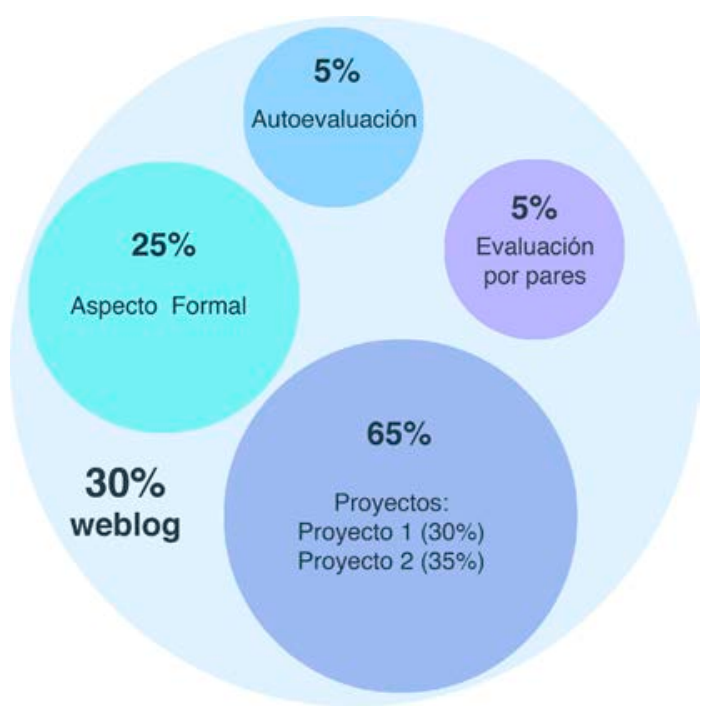

Fig.2. Esquema evaluación weblog

Para la obtención de este $30 \%$ se tienen en cuenta distintos factores con distintas ponderaciones (ver Fig.2):

a) La llamada parte formal con un valor del 25\% evalúa el trabajo individual consistente en: el diseño del edublog, la presentación del contenido, la calidad de la escritura y la revisión ortográfica y gramatical de todo el contenido, así como la búsqueda de información y modelización teórica de las tareas a realizar.

b) La valoración del $65 \%$ se reparte entre los dos proyectos realizados. Con un valor del $30 \%$ se evalúa el trabajo en grupo correspondiente al proyecto 1 y con un $45 \%$ el trabajo en grupo correspondiente al proyecto 2 .

c) Finalmente y a nivel individual con 5\% cada una se evalúa la autoevaluación y la evaluación por pares de los estudiantes. Es de destacar lo críticos que son los estudiantes con su propio trabajo.

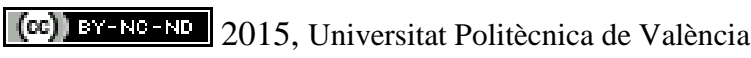

Congreso In-Red (2015) 
Para crear una rúbrica, es necesario dejar claro el propósito de la tarea e identificar qué tarea los estudiantes deben aprender. La rúbrica debe basarse en la finalidad de la tarea de aprendizaje que se evaluará, en consecuencia, es muy importante que el objetivo esté bien definido desde el inicio.

Para el tema específico de álgebra lineal del primer año de Ingeniería en la ETSEIB de la Universidad Politécnica de Cataluña, tal y como hemos dicho anteriormente, se proponen dos rúbricas, la primera para la auto-evaluación de los estudiantes, así como para la evaluación por pares, y la segunda para la evaluación del trabajo del estudiante por parte del docente. La matriz de valoración para evaluar el trabajo de los estudiantes consiste en 3 sub-rúbricas, una para analizar la parte formal del blog del alumno (estructura, ortografía, nivel de idioma, entre otros) y las otras dos para la evaluación de los proyectos que los estudiantes deben realizar.

\section{Resultados}

Con el uso de las diferentes rúbricas dentro del weblog se han mejorado de forma sustancial las calificaciones globales de los alumnos en la asignatura de Álgebra lineal. Estas mejoras se ven reflejadas no sólo en las calificaciones de la asignatura sino en las encuestas realizadas a los alumnos sobre el sistema de evaluación de los trabajos realizados a través del blog. Tal y como se puede ver en los gráficos de la Fig.3, el nivel de comprensión de la asignatura aumentó en global gracias al nuevo planteamiento de la asignatura. La primera gráfica corresponde a la totalidad de los alumnos, la seguna corresponde al grupo de estudiantes 10 en el que el tutor estuvo plenamente implicado en el proyecto de innovación realizado.

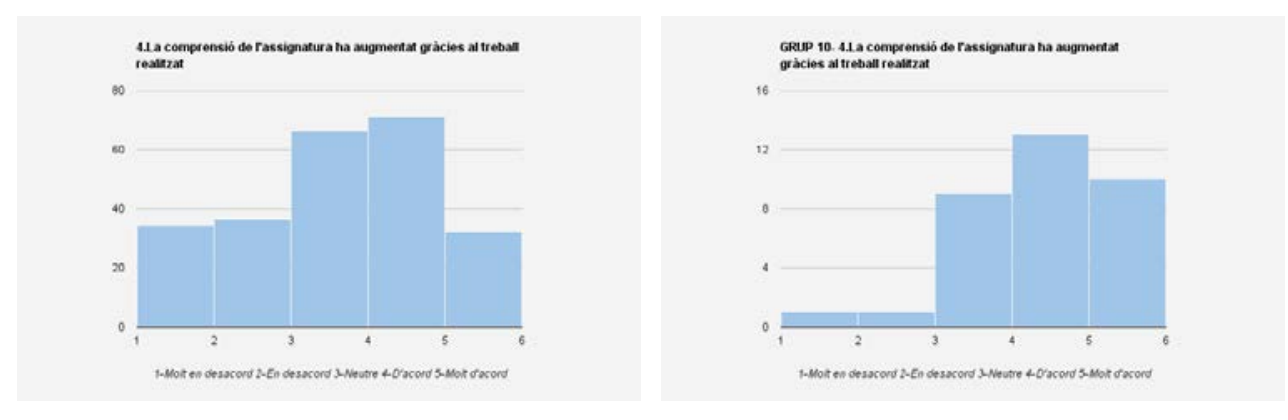

Fig.3. Gráfica encuesta satisfacción

Analizadas todas las preguntas reflexivas que formaban parte de la rúbrica, se ha llegado al siguiente resultado final; de las dos rúbricas y las tres sub-rúbricas realizadas, presentamos a continuación dos ejemplos, una para el profesor y otra para el estudiante. 


\subsection{Rúbrica del profesor}

A continuación se muestra la rúbrica que se ha utilizado para evaluar el trabajo de los estudiantes.

\begin{tabular}{|c|c|c|c|c|c|}
\hline 0 puntos & 1 punto & 2 puntos & 3 puntos & 4 puntos & 5 puntos \\
\hline No presentado & $\begin{array}{l}\text { No ha descrito el } \\
\text { problema } \\
\text { correctamente } \\
\text { porqué no ha } \\
\text { comprendido cual } \\
\text { era la problemática } \\
\text { a solucionar }\end{array}$ & $\begin{array}{l}\text { Ha entendido } \\
\text { el problema, pero } \\
\text { no ha sido capaz } \\
\text { de describir el } \\
\text { problema } \\
\text { correctamente }\end{array}$ & $\begin{array}{l}\text { Ha entendido } \\
\text { el problema y } \\
\text { lo ha descrito } \\
\text { correctamente }\end{array}$ & $\begin{array}{c}\text { Ha descrito el } \\
\text { problema y ha } \\
\text { entendido cual } \\
\text { es la problemática } \\
\text { a solucionar }\end{array}$ & $\begin{array}{l}\text { Ha descrito el } \\
\text { problema perfec- } \\
\text { tamente, ha enten- } \\
\text { dido cual era la } \\
\text { problemática y ha } \\
\text { valorado otros } \\
\text { posibles problemas }\end{array}$ \\
\hline
\end{tabular}

\begin{tabular}{|c|c|c|c|c|c|}
\hline No presentado & $\begin{array}{c}\text { No ha encontrado } \\
\text { la información } \\
\text { necesaria para } \\
\text { solucionar el } \\
\text { problema, y no han } \\
\text { encontrado la fór- } \\
\text { mula adecuada }\end{array}$ & $\begin{array}{c}\text { Ha encontrado } \\
\text { información pero } \\
\text { no ha sido capaz } \\
\text { de modelizar } \\
\text { adecuadamente } \\
\text { para solucionar } \\
\text { el problema }\end{array}$ & $\begin{array}{c}\text { Ha encontrado } \\
\text { información y la } \\
\text { ha modelizado } \\
\text { correctamente }\end{array}$ & $\begin{array}{c}\text { Ha encontrado } \\
\text { información, la } \\
\text { ha modelizado } \\
\text { perfectamente. }\end{array}$ & $\begin{array}{c}\text { Ha encontrado } \\
\text { información, la ha } \\
\text { modelizado perfec- } \\
\text { tamente y ha des- } \\
\text { crito los pasos de } \\
\text { como ha llegado } \\
\text { a la conclusión }\end{array}$ \\
Búsqueda de la información y modelización & & & \\
\hline
\end{tabular}

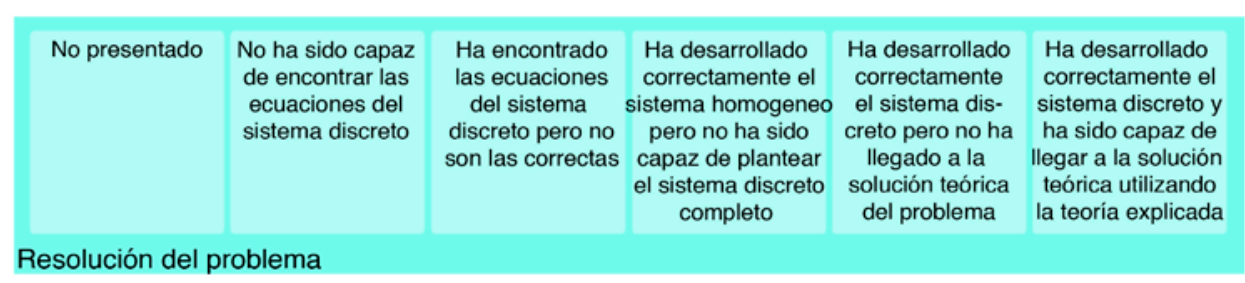

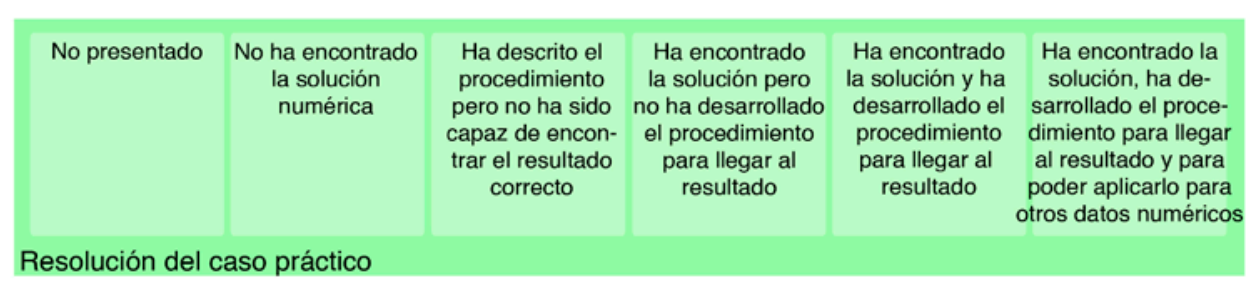

$\begin{array}{cccccc}\text { No presentado } & \begin{array}{c}\text { No ha hecho una } \\ \text { valoración } \\ \text { critica adecuada } \\ \text { del problema que } \\ \text { ha resuelto }\end{array} & \begin{array}{c}\text { Ha valorado de } \\ \text { forma crítica la } \\ \text { resolución del } \\ \text { problema pero } \\ \text { con algun error } \\ \text { en la misma }\end{array} & \begin{array}{c}\text { Ha valorado } \\ \text { correctamente } \\ \text { la resolución del } \\ \text { problema }\end{array} & \begin{array}{c}\text { Ha valorado } \\ \text { de forma crítica } \\ \text { la resolución } \\ \text { del problema } \\ \text { perfectamente }\end{array} & \begin{array}{c}\text { Ha valorado de } \\ \text { forma crítica la } \\ \text { resolución del } \\ \text { problema perfec- } \\ \text { tamente y lo ha } \\ \text { relacionado con } \\ \text { otros problemas }\end{array} \\ \end{array}$

Análisis crítico del resultado

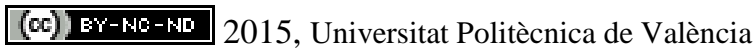

Congreso In-Red (2015) 
El uso de weblogs en la educación superior y su evaluación mediante rúbrica

Fig4. Rúbrica de profesor

\subsection{Rúbrica del estudiante}

A continuación se muestra la rúbrica que se ha utilizado para la autoevaluación y la evaluación por pares del trabajo de los estudiantes.

\begin{tabular}{|c|c|c|}
\hline Insuficiente & Suficiente & Buen trabajo \\
\hline $\begin{array}{l}\text { El weblog de mis compañeros } \\
\text { no tiene demasiada estructura } \\
\text { ni coherencia }\end{array}$ & $\begin{array}{l}\text { El weblog de mis compañeros } \\
\text { está bien ordenado y tiene una } \\
\text { estructura coherente }\end{array}$ & $\begin{array}{l}\text { El weblog de mis compañeros } \\
\text { está perfectamente organizado } \\
\text { y tiene una estructura coherente. } \\
\text { Han tratado todos los detalles } \\
\text { de la página }\end{array}$ \\
\hline
\end{tabular}

\begin{tabular}{|l|c|c|}
\hline $\begin{array}{c}\text { Han cometido algunos errores } \\
\text { ortográficos y gramaticales ya } \\
\text { que no han revisado con } \\
\text { cuidado el texto }\end{array}$ & $\begin{array}{c}\text { Han cometido algun error } \\
\text { ortográfico, más a nivel } \\
\text { tipográfico. El texto está bien } \\
\text { redactado y es comprensible }\end{array}$ & $\begin{array}{c}\text { Han redactado el texto sin } \\
\text { errores ortográficos ni } \\
\text { gramaticales. Han revisado } \\
\text { con cuidado la redacción } \\
\text { y es comprensible del todo }\end{array}$ \\
\hline Calidad de escritura y revisión ortográfica y gramatical & \\
\hline
\end{tabular}

\begin{tabular}{|l|c|c|c|}
$\begin{array}{c}\text { Han encontrado información } \\
\text { pero han tenido dificultades } \\
\text { para modelizar los problemas } \\
\text { sin ayuda no lo hubieran } \\
\text { conseguido }\end{array}$ & $\begin{array}{c}\text { Han encontrado junto con sus } \\
\text { compañeros de equipo, } \\
\text { información y han conseguido } \\
\text { modelizar correctamente los } \\
\text { problemas (mínimo uno de ellos) }\end{array}$ & $\begin{array}{c}\text { Han encontrado información, } \\
\text { han modelizado perfectamente } \\
\text { y han descrito los pasos de } \\
\text { como han llegado a encontrar } \\
\text { los modelos }\end{array}$ \\
\begin{tabular}{c} 
Búsqueda de información y modelización \\
\hline
\end{tabular}
\end{tabular}
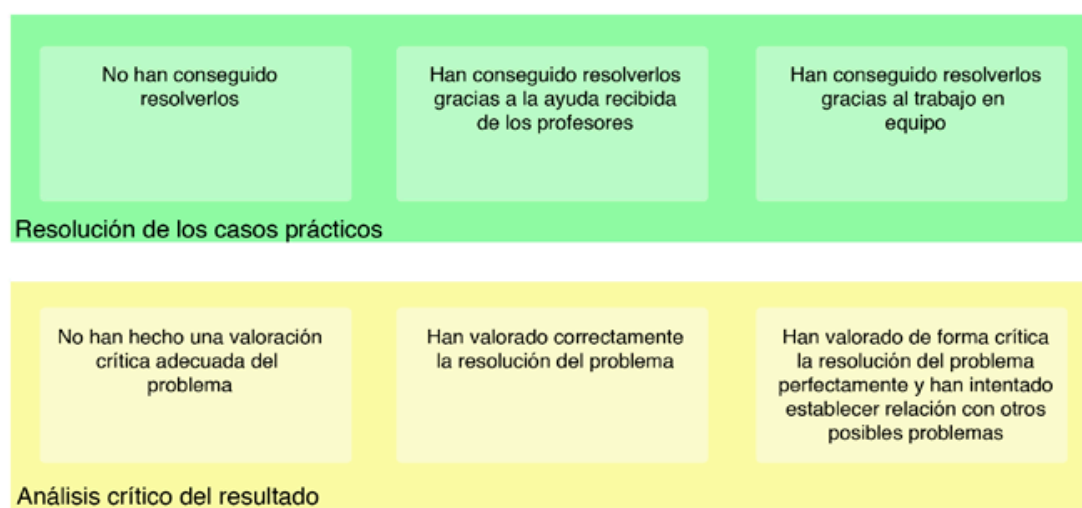

Fig.5. Rúbrica del estudiante

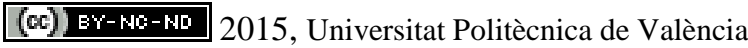

Congreso IN-RED (2015) 


\section{Conclusiones}

El estudiante valora de forma muy positiva el disponer desde el inicio del contenido de la rúbrica, mostrando de forma detallada que aspectos muestran una buena comprensión de los contenidos y garantizan una buena evolución en el aprendizaje de la materia en cuestión.

\section{Referencias}

ALLAN, M. (2004). Assessing academic programs in higher education. Bolton, MA: Anker.

BLOOM, B.S., MADAUS, G.F., y HASTINGS, J.T. (1981). "Evaluation to improve learning”. <http://agris.fao.org/agrissearch/search.do?recordID=US201300389689> [Consulta: 4 de mayo de 2015]

DOMÍNGUEZ, S., GARCÍA-PLANAS, M.I., TABERNA, J. y PALAU, R." Evaluating an E-portfolio for a Linear Algebra". en: INTED2015 Proceedings. p. 1366-1372.

KOH, J.H.L. (2013). “A rubric for assessing teachers' lesson activities with respect to TPACK for meaningful learning with ICT". Australasian Journal of Educational Technology, vol. 29, issue 6, p. 887-900.

FALCHIKO, N. y GOLFINCH, J. (2000). "Student Peer Assessment in Higher Education: A Meta-Analysis Comparing Peer and Teacher Marks". Review of Educational Research, vol. 70, p. 287.

HERNÁNDEZ SÁNCHEZ, A.A., (2010). “Necesidad de la evaluación en el proceso de aprendizaje" monografías.com

MOSKA, B. (2000). "Scoring Rubrics: What, When and How? Practical Assessment", Research \& Evaluation, vol. 7, issue 3.

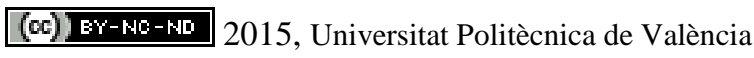

Congreso In-Red (2015) 